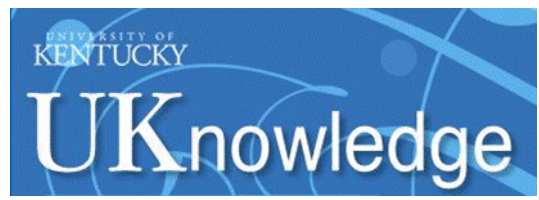

University of Kentucky

UKnowledge

\title{
Thiamine Deficiency Induces Endoplasmic Reticulum Stress and Oxidative Stress in Human Neurons Derived from Induced Pluripotent Stem Cells
}

Xin Wang

University of Kentucky, xin.wang3@uky.edu

Mei Xu

University of Kentucky, mxu222@uky.edu

Jacqueline A. Frank

University of Kentucky, j.frank@uky.edu

Zun-Ji Ke

Shanghai University of Traditional Chinese Medicine, China

Jia Luo

University of Kentucky, jialuo888@uky.edu

Follow this and additional works at: https://uknowledge.uky.edu/pharmacol_facpub

Part of the Cell and Developmental Biology Commons, and the Pharmacology, Toxicology and Environmental Health Commons

Right click to open a feedback form in a new tab to let us know how this document benefits you.

\section{Repository Citation}

Wang, Xin; Xu, Mei; Frank, Jacqueline A.; Ke, Zun-Ji; and Luo, Jia, "Thiamine Deficiency Induces Endoplasmic Reticulum Stress and Oxidative Stress in Human Neurons Derived from Induced Pluripotent Stem Cells" (2017). Pharmacology and Nutritional Sciences Faculty Publications. 90.

https://uknowledge.uky.edu/pharmacol_facpub/90

This Article is brought to you for free and open access by the Pharmacology and Nutritional Sciences at UKnowledge. It has been accepted for inclusion in Pharmacology and Nutritional Sciences Faculty Publications by an authorized administrator of UKnowledge. For more information, please contact UKnowledge@lsv.uky.edu. 


\title{
Thiamine Deficiency Induces Endoplasmic Reticulum Stress and Oxidative Stress in Human Neurons Derived from Induced Pluripotent Stem Cells
}

\author{
Digital Object Identifier (DOI) \\ https://doi.org/10.1016/j.taap.2017.02.009 \\ Notes/Citation Information \\ Published in Toxicology and Applied Pharmacology, v. 320, p. 26-31. \\ (C) 2017 Elsevier Inc. All rights reserved.
}

This manuscript version is made available under the CC-BY-NC-ND 4.0 license https://creativecommons.org/licenses/by-nc-nd/4.0/.

The document available for download is the author's post-peer-review final draft of the article. 
Toxicol Appl Pharmacol. 2017 April 01; 320: 26-31. doi:10.1016/j.taap.2017.02.009.

\title{
Thiamine deficiency induces endoplasmic reticulum stress and oxidative stress in human neurons derived from induced pluripotent stem cells
}

\author{
Xin Wang ${ }^{\mathrm{a}}$, Mei Xü ${ }^{\mathrm{a}}$, Jacqueline A. Frank ${ }^{\mathrm{a}}$, Zun-ji Ke ${ }^{\mathrm{b}}$, and Jia Luo ${ }^{\mathrm{a}, \mathrm{b},{ }^{*}}$ \\ aDepartment of Pharmacology and Nutritional Sciences, University of Kentucky College of \\ Medicine, Lexington, KY 40536, United States \\ ${ }^{b}$ Department of Biochemistry, Shanghai University of Traditional Chinese Medicine, Shanghai, \\ China 201203
}

\section{Abstract}

Thiamine (vitamin B1) deficiency (TD) plays a major role in the etiology of Wernicke's encephalopathy (WE) which is a severe neurological disorder. TD induces selective neuronal cell death, neuroinflammation, endoplasmic reticulum (ER) stress and oxidative stress in the brain which are commonly observed in many aging-related neurodegenerative diseases, such as Alzheimer's disease (AD), Parkinson's disease (PD), Huntington's disease (HD) and progressive supranuclear palsy (PSP). However, the underlying cellular and molecular mechanisms remain unclear. The progress in this line of research is hindered due to the lack of appropriate in vitro models. The neurons derived for the human induced pluripotent stem cells (hiPSCs) provide a relevant and powerful tool for the research in pharmaceutical and environmental neurotoxicity. In this study, we for the first time used human induced pluripotent stem cells (hiPSCs)-derived neurons (iCell neurons) to investigate the mechanisms of TD-induced neurodegeneration. We showed that TD caused a concentration- and duration-dependent death of iCell neurons. TD induced ER stress which was evident by the increase in ER stress markers, such as GRP78, XBP-1, CHOP, ATF-6, phosphorylated eIF2a, and cleaved caspase-12. TD also triggered oxidative stress which was shown by the increase in the expression 2,4-dinitrophenyl (DNP) and 4-hydroxynonenal (HNE). ER stress inhibitors (STF-083010 and salubrinal) and antioxidant $N$ acetyl cysteine (NAC) were effective in alleviating TD-induced death of iCell neurons, supporting the involvement of ER stress and oxidative stress. It establishes that the iCell neurons are a novel tool to investigate cellular and molecular mechanisms for TD-induced neurodegeneration.

\footnotetext{
"Correspondence author at: Department of Pharmacology and Nutritional Sciences, University of Kentucky College of Medicine, 132 Health Sciences Research Building, 1095 Veterans Drive, Lexington, Kentucky 40536, United States. jialuo888@uky.edu (J. Luo). Conflict of interest

All authors have read and approved the manuscript. We do not have conflict of interest. This manuscript has not been and will not be submitted or published in other scientific journals in whole or in part while it is under the consideration of Toxicol Appl Pharmacol.

Transparency document

The Transparency document associated with this article can be found, in the online version.
} 


\section{Keywords}

Endoplasmic reticulum stress; Neurodegeneration; Reactive oxygen species; Stem cells; Vitamins

\section{Introduction}

Thiamine (vitamin B1) deficiency (TD) causes Wernicke's encephalopathy (WE) which is a neuropsychiatric syndrome characterized by opthalmoplegia, ataxia and memory loss (Rao et al., 1996; Todd and Butterworth, 1999; Ke et al., 2003). Brain tissues isolated from WE patients display gliosis, vascular damage, and neuronal loss in specific regions, such as the mammillary bodies, inferior olive, and thalamus (Troncoso et al., 1981; Kril, 1996; Gibson et al., 1999; Pannunzio et al., 2000). However, the cellular and molecular mechanisms underlying TD-induced neuronal damage remain unclear. In experimental models, which include in vitro neuronal cultures and animal models, TD induces selective neuronal cell death, neuroinflammation, glial activation, endoplasmic reticulum (ER) stress and oxidative stress in the central nervous system (CNS). These features are commonly observed in many aging-related neurodegenerative diseases, such as Alzheimer's disease (AD), Parkinson's disease (PD), Huntington's disease (HD) and progressive supranuclear palsy (PSP) (Ke and Gibson, 2004). Therefore, experimental TD models are useful not only for studying the mechanisms of WE but also for that of neurodegeneration in aging-associated degenerative diseases (Schwab et al., 1996; Gibson and Zhang, 2001; Park et al., 2001).

Up to date, in vitro neuronal models, mainly rodent primary neuron cultures, have made significant contributions to our understanding of the mechanisms of TD's effects on neurons because they have many advantages in investigating cellular and molecular events (Wang et al., 2007a; Lee et al., 2010; Cruz et al., 2012). However, these rodent-derived in vitro models may not be an appropriate reflection of native human neurons and their physiological relevance is questionable. The discovery of human induced pluripotent stem cells (hiPSCs) has been revolutionizing the research in neurotoxicity because these cells can be differentiated into neurons, providing a more biologically relevant model. For example, the hiPSCs obtained from AD patients may offer a good model to investigate the interplay of genetics and environmental factors in the context of $\mathrm{AD}$ pathogenesis. The neurons derived from hiPSCs are therefore a novel and powerful tool to study the genetics, cellular/molecular biology of human neurons in response to environmental impacts or neurotoxins. In this study, we describe for the first time the use of hiPSCs-derived neurons (iCell neurons) as a new platform for studying the mechanisms of TD-induced neurodegeneration. The iCell neurons were differentiated and cryopreserved by Cellular Dynamics International (Madison, WI) and consist of a purified pan-neuronal population comprised predominantly of gamma aminoisobutyric acid (GABA)ergic and glutamatergic neurons (Whitemarsh et al., 2012). We show here that TD causes death of iCell neurons and induces ER stress and oxidative stress. The inhibition of ER stress or oxidative stress significantly reduces TDinduced cell death. This study establishes that the iCell neurons are a novel and useful tool to investigate cellular and molecular mechanisms for TD-induced CNS damage. 


\section{Materials and methods}

\subsection{Reagents}

Poly-D-lysine hydrobromide, amprolium, and laminin were purchased from Sigma (St. Louis, MO, USA). Rabbit anti-p-eIF2a, anti-caspase-12, anti-ATF6, anti-dinitrophenol (DNP) antibodies were obtained from Cell signaling Technology (Danvers, MA). Rabbit anti-GRP78 antibody was obtained from Novus Biologicals (Littleton, CO). Mouse antiCHOP antibody was obtained from Thermo Fisher Scientific (Rockford, IL). Rabbit anti-4hydroxynonenal (HNE) antibody was obtained from lifespan BioSciences (Seattle, WA). HRP-conjugated anti-rabbit and anti-mouse secondary antibodies were purchased from GE Healthcare life Sciences (Piscataway, NJ). Calcein AM was obtained from Thermo Fisher Scientific (Waltham, MA).

\subsection{Culture of human neurons derived from induced pluripotent stem cells}

Human neurons differentiated from induced pluripotent stem (iPS) cells (iCell neurons) were purchased from Cellular Dynamics International (Madison, WI, USA) and stored in frozen vials containing at least 2.5 million cells. These cells display characteristics of primary forebrain neurons and are widely used in the scientific community for cellular, molecular and electrophysiological studies (Chai et al., 2012; Haythornthwaite et al., 2012; Whitemarsh et al., 2012; Xu et al., 2013; Dage et al., 2014). Cells were handled according to the instructions provided by the manufacturer. Briefly, iCell neurons were removed from liquid nitrogen storage and thawed for $3 \mathrm{~min}$ in a $37^{\circ} \mathrm{C}$ water bath. Cells were gently transferred to a $50 \mathrm{ml}$ tube and $1 \mathrm{ml}$ of the maintenance medium (provided by the manufacturer) was added drop-wise. Cells were swirled gently to minimize osmotic shock and an additional $8 \mathrm{ml}$ of medium was slowly added. Cells were plated at a density of $4 \times$ $10^{4} /$ well on 96 -well plates, $9 \times 10^{4} /$ well on 24 -well plate, or $2 \times 10^{5} /$ dish on 60 -mm cell culture dishes. Plates or cell culture dishes were pre-coated with poly-L-ornithine (PLO) $(0.01 \%)$ followed by a layer of laminin $(3.3 \mu \mathrm{g} / \mathrm{ml})$ on top. Cells were cultured in the maintenance medium at $37{ }^{\circ} \mathrm{C}$ in $5 \% \mathrm{CO}_{2}$. The medium was changed after $24 \mathrm{~h}$, and then every other day thereafter until the start of the experiment. For immunocytochemistry (IHC) studies, iCell neurons $\left(8 \times 10^{4} /\right.$ chamber $)$ were cultured on glass chamber slides pre-coated with POL/laminin.

\subsection{Cytotoxicity assessment}

Cell viability was determined by CCK-8 kit which measures the activity of mitochondrial dehydrogenase (Dojindo, MD, USA). Briefly, $10 \mu \mathrm{l}$ of CCK-8 solution was added the cultures and incubated for 2-3 h. The activity of mitochondrial dehydrogenase was determined by the color product, formazan which was measured at an absorbance of $450 \mathrm{~nm}$ using a microplate reader. In addition, cell viability and morphology were also determined by calcein AM labeling. Calcein AM is able to cross the cell membrane and is metabolized in the cytosol to produce fluorescent calcein by cytosolic esterases. Dead cells are either unable to metabolize calcein AM or retain fluorescent calcein. The cultures were treated with $1 \mu \mathrm{M}$ calcein $\mathrm{AM}$ for $30 \mathrm{~min}$ at $37^{\circ} \mathrm{C}$ in $5 \% \mathrm{CO}_{2}$, and then examined under a fluorescent microscope. 


\subsection{Immunoblotting}

The procedure for immunoblotting has been previously described (Ke and Gibson, 2004; Wang et al., 2007a). Briefly, aliquots of proteins ( $30 \mu \mathrm{g})$ were loaded into the lanes of a sodium dodecyl sulfate-polyacrylamide gel. The proteins were separated by electrophoresis and transferred to nitrocellulose membranes. The membranes were blocked with 5\% BSA in $0.01 \mathrm{M}$ Tris-buffered saline (TBS) (pH 7.4) and 0.05\% Tween-20 (TBST) at room temperature for $1 \mathrm{~h}$. Then, the membranes were incubated with primary antibodies directed against target proteins overnight at $4{ }^{\circ} \mathrm{C}$. The final dilutions for primary antibodies were: GRP78, 1:1,000; XBP-1, 1:1,000; p-eIF2a, 1:1,000; Chop, 1:500; caspase-12, 1:1,000; HNE, 1:1,000; DNP, 1:1,000; and actin, 1:5,000. After two quick washes in TBST, the membranes were incubated with secondary antibodies conjugated to horseradish peroxidase (Amersham, Arlington, Heights, IL) diluted at 1:5000 in TBST containing 5\% BSA for $1 \mathrm{~h}$. The immunocomplexes were detected by the enhanced chemiluminescence method (Amersham). The density of immunoblotting was quantified with the software of ImageJ (version 1.48; National Institutes of Health, Bethesda, MD).

\subsection{Statistical analysis}

The data were expressed as mean \pm SEM. Statistical significance was determined by the one-way ANOVA followed by Scheffé's post-doc test at a significance level of 5\%. The analyses were performed using SPSS software (SPSS, Chicago, IL, USA).

\section{Results}

\subsection{TD induces death of iCell neurons}

Amprolium is a thiamine antagonist and widely used in vitro and in vivo to induce thiamine deficiency (Wang et al., 2007a). We examined the effect of amprolium on the viability of iCell neurons by CCK-8 assay and calcein AM staining (Fig. 1). Amprolium induced a concentration- and duration-dependent decrease of the viability of iCell neurons. At 1 and 2 $\mathrm{mM}$, amprolium significantly reduced cell viability by $16 \%$ and $40 \%$ respectively after 5 days of exposure, and by $36 \%$ and $46 \%$, respectively following 7 days of exposure (Fig. 1A). Amprolium at the lower concentration, $0.5 \mathrm{mM}$, also reduced cell viability, but took a longer time; it decreased cell viability by $15 \%$ after 7 days of exposure. It is interesting to note that at $0.5 \mathrm{mM}, 3-5$ days of exposure slightly increased cell viability (Fig. 1A). We used calcein AM staining to further determine the cell viability and morphology. Amprolium not only reduced the number of viable cells but also caused the loss of neurites in the iCell neurons (Fig. 1B).

\subsection{TD causes ER stress and oxidative stress in iCell neurons}

Since ER stress and oxidative stress play an important role in TD-induced neurodegeneration (Liu et al., 2016), we first sought to determine whether amprolium induced ER stress in iCell neurons. As shown in Fig. 2, amprolium significantly increased the expression of a number of markers for ER stress, such as GRP78, XBP-1, CHOP, peIF2a, cleaved caspase-12 and ATF6. Amprolium-induced ER stress occurred earlier than cell death; generally, amprolium-induced upregulation of ER stress markers was evident 1 
day after amprolium treatment. For GRP78, spliced XBP-1 and phosphorylation of eIF2a, amprolium induced a drastic increase in their expression after 1-2 days of exposure, and then the increase diminished thereafter. For ATF-6, an amprolium-induced increase was observed after 2 days of exposure and the increase sustained for 3 days, then diminished thereafter. For CHOP and cleaved caspase-12, amprolium increased their expression after 1 day of exposure and the increase sustained for at least 4 days.

To determine whether amprolium induced oxidative stress in iCell neurons, we examined the expression of 4-hydroxynonenal (4-HNE) and 2,4-dinitrophenol (DNP) which are reliable biomarkers for lipid peroxidation and protein oxidation, respectively (Perluigi et al., 2012). As shown in Fig. 3, amprolium significantly increased the expression of 4-HNE and DNP in iCell neurons. Amprolium (1 mM) significantly increased the expression of 4-HNE and DNP after 1 day of exposure and the increase was sustained for 3 days, and then diminished thereafter.

\subsection{Alleviating ER and oxidative stress reduces TD neurotoxicity}

To determine whether ER stress and oxidative stress contributed to TD-induced death of iCell neurons, we inhibited TD-induced ER stress and oxidative stress using specific inhibitors and antioxidants. STF083010 and salubrinal have been used to experimentally alleviate ER stress. STF083010 is a novel small-molecule inhibitor of Ire1 (Papandreou et al., 2011). Salubrinal selectively inhibits eIF2a dephosphorylation and is shown to protect cells against ER stress-mediated apoptosis (Cnop et al., 2007). $\mathrm{N}$-acetyl Cysteine (NAC) is a thiol-containing antioxidant and potent free radical scavenger (Ates et al., 2008). As shown in Fig. 4A, pretreatment with STF083010 $(10 \mu \mathrm{M})$ or salubrinal $(10 \mu \mathrm{M})$ significantly mitigated amprolium-induced death of iCell neurons. On the other hand, NAC completely eliminated TD-induced cell death. This result was confirmed by the study of calcein AM staining which showed that STF083010 and NAC alleviated TD-induced cytotoxicity in iCell neurons. Together these data indicate that ER stress and oxidative stress are indeed involved in TD-induced neurotoxicity.

\section{Discussion}

The understanding of the cellular and molecular mechanisms underlying TD-induced neurotoxicity and aging-associated neurodegeneration has been hindered by the lack of relevant in vitro models. Here we describe for the first time the use of the neurons derived from human induced pluripotent stem cells (iCell neurons) to investigate the mechanisms of TD-induced neurodegeneration. We show that TD causes a concentration- and durationdependent death of iCell neurons. TD induces ER stress as well as oxidative stress and alleviating ER stress/ oxidative stress is sufficient to mitigate TD-induced death of iCell neurons, indicating the involvement of ER stress and oxidative stress in TD neurotoxicity.

iCell neurons developed by Cellular Dynamics International resembles neurons in neonatal prefrontal cortex. They express functional ionotropic glutamate [a-amino-3-hydroxy-5methyl-4-isoxazolepropionic acid (AMPA) and $N$-methyl-D-aspartate (NMDA)] receptors, gamma-aminobutyric acid type $\mathrm{A}\left(\mathrm{GABA}_{\mathrm{A}}\right)$ receptor, voltage-gated sodium and calcium channels, and nicotinic acetylcholine receptors (nAChRs) (Dage et al., 2014; Chatzidaki et 
al., 2015). These cells provide a biologically relevant human cell model for the assessment of pharmaceutical and environmental neurotoxicity as well as the investigation of neurodegenerative diseases. For example, iCell Neurons were employed to study the processing and cellular trafficking of tau, a protein that plays an important role in the development of AD and other neurodegenerative disorders (Chai et al., 2012). iCell Neurons were successfully used in a high-throughput small molecule screen for identifying potential protective molecules that can alleviate neurodegeneration associated with $\mathrm{AD}$ (Xu et al., 2013). iCell neurons exhibited toxicity to a-synuclein and were used as an in vitro PD model. The potential neuroprotective effect of thymoquinone (TQ) on a-synuclein-induced alteration in synapse density, synaptic vesicle recycling and electrical activity was assessed in iCell neurons (Alhebshi et al., 2014).

Amprolium is a competitive inhibitor of thiamine transport and has been widely used to deplete intracellular thiamine. We show here that amprolium at $\mathrm{mM}$ range caused the death of human iCell neurons. This finding is consistent with previous studies carried out by ourselves and other investigators showing that in cultured neuronal cells the effective concentrations of amprolium are at mM range (Wang et al., 2007b; Chornyy and Parkhomenko, 2008; Bizon-Zygmanska et al., 2011; Liu et al., 2016). Alternatively, future studies using other thiamine antagonists, such as pyrithiamine, may be considered. It is interesting to note that amprolium slightly increases cell survival at $0.5-1 \mathrm{mM}$. The underlying mechanisms are currently unknown. It is possible that amprolium may initially activate some cytoprotective programs, such as autophagy.

TD induces ER stress in iCell neurons. ER is the largest organelle regulating protein folding, modification and transport. It also processes the synthesis and distribution of phospholipids and steroids. ER is the site of calcium ion storage and regulates intracellular calcium homeostasis (Schroder, 2008; Bravo et al., 2013). Alterations in ER homeostasis, such as increased protein synthesis, accumulation of misfolded proteins, or alterations in the calcium or redox balance, lead to ER stress (Ozcan and Tabas, 2012). The cellular adaptive response to cope with ER stress is known as the unfolded protein response (UPR). Initially, the UPR functions to restore ER homeostasis by increasing protein folding capacity and decreasing the accumulation of unfolded proteins. However, when UPR is insufficient to restore ER homeostasis, apoptosis occurs (Morishima et al., 2004; Tabas and Ron, 2011). During the UPR, three major UPR sensors, namely PERK (protein kinase RNA-like ER kinase), IRE1 (inositol requiring protein-1), and ATF6 (activating transcription factor-6), are activated to restore folding capacity (Hetz et al., 2011; Yang and Luo, 2015). Neurodegenerative diseases, such as $\mathrm{AD}, \mathrm{PD}$, and $\mathrm{HD}$, have in common the presence of protein aggregates in specific brain areas where significant neuronal loss is detected. Emerging evidence suggests that ER stress may contribute to the neurodegeneration (Perri et al., 2016).

Oxidative stress is caused by the disequilibrium between reactive oxygen species (ROS) production and the ability to detoxify the ROS (Fischer and Maier, 2015). The brain is particularly vulnerable to oxidative stress due to its high oxygen consumption and high production of reactive oxygen and nitrogen species. Accumulating evidence has demonstrated that oxidative stress plays an important role in excitotoxicity, axonal damage and neuronal death (Chiurchiu et al., 2015), and is involved in the onset and progression of 
several neurodegenerative diseases, such as AD, PD, HD, and amyotrophic lateral sclerosis (ALS) (Radi et al., 2014; Cobb and Cole, 2015). Oxidative stress also plays an important role in TD-induced brain damage (Liu et al., 2016). However, the mechanisms underlying TD-induced oxidative stress are unclear. The current study indicates that iCell neurons are a good model to investigate TD-induced oxidative cells. There is considerable interaction between oxidative stress and ER stress.

The cellular/molecular mechanisms by which amprolium causes ER stress and oxidative stress are currently unclear. For inducing ER stress, amprolium may causes a disruption of intracellular calcium homeostasis or impair the ER chaperons which are responsible for protein folding. For inducing oxidative stress, amprolium may disrupt the antioxidant system or directly cause mitochondrial damage (Liu et al., 2016). The effect of amprolium is complex and both direct and indirect mechanisms may be involved. There is considerable cross talk between oxidative stress and ER stress. Usually oxidative stress promotes ER stress but sometimes ER stress can cause oxidative stress as well (Zhang, 2010). Amprolium-induced ER stress may also be promoted by oxidative stress.

Recent findings suggest that TD can induce autophagy in neurons (Meng et al., 2013; Liu et al., 2016). Autophagy is a cellular catabolic process for the degradation of cytoplasmic components through lysosomes (Levine and Klionsky, 2004; Mizushima and Klionsky, 2007). Autophagy is fundamental for the maintenance of homeostasis and especially important in post-mitotic neurons. Both oxidative stress and ER stress may activate autophagy (Luo, 2014). Therefore, ER stress, oxidative stress, and autophagy are interconnected and interdependent processes. In normal neurons, the homeostasis of these dynamic processes is well controlled and maintained. However, TD disrupts the homeostasis, eventually causing neurodegeneration in the CNS. We propose that it is the interplay of ER stress, oxidative stress, and autophagy that contributes to TD-induced neurodegeneration (Liu et al., 2016). Our future studies need to determine whether TD causes autophagy. Therefore, iCell neurons may also provide a powerful tool to investigate in interaction among ER stress, oxidative stress and autophagy in the context of TD-induced neurodegeneration.

\section{Acknowledgments}

This work was supported by grants from the National Institutes of Health (NIH) (AA017226 and AA015407). It is also supported in part by the Department of Veterans Affairs, Veterans Health Administration, Office of Research and Development (Biomedical Laboratory Research and Development: BX001721).

\section{Abbreviations}
AD
Alzheimer's disease
ATF6 activating transcription factor 6
CHOP transcriptional factor C/EBP homologous protein
DNP dinitrophenol
ER endoplasmic reticulum 


$\begin{array}{ll}\text { EIF2a } & \text { eukaryotic initiation factor } 2 a \\ \text { HD } & \text { Huntington's disease } \\ \text { HNE } & \text { 4-hydroxynonenal } \\ \text { hiPSCs } & \text { human induced pluripotent stem cells } \\ \text { NAC } & \text { N-acetyl cysteine NAC } \\ \text { PD } & \text { Parkinson's disease } \\ \text { PERK } & \text { protein kinase R-like endoplasmic reticulum kinase } \\ \text { PSP } & \text { progressive supranuclear palsy } \\ \text { ROS } & \text { reactive oxygen species } \\ \text { TD } & \text { thiamine deficiency } \\ \text { UPR } & \text { unfolded protein response } \\ \text { XBP1 } & \text { X-box binding protein-1 }\end{array}$

\section{References}

Alhebshi AH, Odawara A, Gotoh M, Suzuki I. Thymoquinone protects cultured hippocampal and human induced pluripotent stem cells-derived neurons against alpha-synuclein-induced synapse damage. Neurosci Lett. 2014; 570:126-131. [PubMed: 24080376]

Ates B, Abraham L, Ercal N. Antioxidant and free radical scavenging properties of $N$-acetylcysteine amide (NACA) and comparison with $N$-acetylcysteine (NAC). Free Radic Res. 2008; 42:372-377. [PubMed: 18404536]

Bizon-Zygmanska D, Jankowska-Kulawy A, Bielarczyk H, Pawelczyk T, Ronowska A, Marszall M, Szutowicz A. Acetyl-CoA metabolism in amprolium-evoked thiamine pyrophosphate deficits in cholinergic SN56 neuroblastoma cells. Neurochem Int. 2011; 59:208-216. [PubMed: 21672592]

Bravo R, Parra V, Gatica D, Rodriguez AE, Torrealba N, Paredes F, Wang ZV, Zorzano A, Hill JA, Jaimovich E, Quest AF, Lavandero S. Endoplasmic reticulum and the unfolded protein response: dynamics and metabolic integration. Int Rev Cell Mol Biol. 2013; 301:215-290. [PubMed: 23317820]

Chai X, Dage JL, Citron M. Constitutive secretion of tau protein by an unconventional mechanism. Neurobiol Dis. 2012; 48:356-366. [PubMed: 22668776]

Chatzidaki A, Fouillet A, Li J, Dage J, Millar NS, Sher E, Ursu D. Pharmacological characterisation of nicotinic acetylcholine receptors expressed in human iPSC-derived neurons. PLoS One. 2015; 10:e0125116. [PubMed: 25906356]

Chiurchiu V, Orlacchio A, Maccarrone M. Is modulation of oxidative stress an answer? The state of the art of redox therapeutic actions in neurodegenerative diseases. Oxidative Med Cell Longev. 2015; 2016:7909380.

Chornyy SA, Parkhomenko YM. Comparative characteristic of thiamine antagonists on apoptosis induction in different types of nerve cell lines. Ukr Biokhim Zh. 2008; 80:76-84.

Cnop M, Ladriere L, Hekerman P, Ortis F, Cardozo AK, Dogusan Z, Flamez D, Boyce M, Yuan J, Eizirik DL. Selective inhibition of eukaryotic translation initiation factor 2 alpha dephosphorylation potentiates fatty acid-induced endoplasmic reticulum stress and causes pancreatic beta-cell dysfunction and apoptosis. J Biol Chem. 2007; 282:3989-3997. [PubMed: 17158450]

Cobb CA, Cole MP. Oxidative and nitrative stress in neurodegeneration. Neurobiol Dis. 2015; 84:421. [PubMed: 26024962] 
Cruz JS, Kushmerick C, Moreira-Lobo DC, Oliveira FA. Thiamine deficiency in vitro accelerates Atype potassium current inactivation in cerebellar granule neurons. Neuroscience. 2012; 221:108114. [PubMed: 22771620]

Dage JL, Colvin EM, Fouillet A, Langron E, Roell WC, Li J, Mathur SX, Mogg AJ, Schmitt MG, Felder CC, Merchant KM, Isaac J, Broad LM, Sher E, Ursu D. Pharmacological characterisation of ligand- and voltage-gated ion channels expressed in human iPSC-derived forebrain neurons. Psychopharmacology. 2014; 231:1105-1124. [PubMed: 24429870]

Fischer R, Maier O. Interrelation of oxidative stress and inflammation in neurodegenerative disease: role of TNF. Oxidative Med Cell Longev. 2015; 2015:610813.

Gibson GE, Zhang H. Abnormalities in oxidative processes in non-neuronal tissues from patients with Alzheimer's disease. J Alzheimers Dis. 2001; 3:329-338. [PubMed: 12214054]

Gibson GE, Park LC, Zhang H, Sorbi S, Calingasan NY. Oxidative stress and a key metabolic enzyme in Alzheimer brains, cultured cells, and an animal model of chronic oxidative deficits. Ann N Y Acad Sci. 1999; 893:79-94. [PubMed: 10672231]

Haythornthwaite A, Stoelzle S, Hasler A, Kiss A, Mosbacher J, George M, Bruggemann A, Fertig N. Characterizing human ion channels in induced pluripotent stem cell-derived neurons. J Biomol Screen. 2012; 17:1264-1272. [PubMed: 22923790]

Hetz C, Martinon F, Rodriguez D, Glimcher LH. The unfolded protein response: integrating stress signals through the stress sensor IRE1alpha. Physiol Rev. 2011; 91:1219-1243. [PubMed: 22013210]

Ke ZJ, Gibson GE. Selective response of various brain cell types during neurodegeneration induced by mild impairment of oxidative metabolism. Neurochem Int. 2004; 45:361-369. [PubMed: 15145550]

Ke ZJ, DeGiorgio LA, Volpe BT, Gibson GE. Reversal of thiamine deficiency-induced neurodegeneration. J Neuropathol Exp Neurol. 2003; 62:195-207. [PubMed: 12578229]

Kril JJ. Neuropathology of thiamine deficiency disorders. Metab Brain Dis. 1996; 11:9-17. [PubMed: 8815394]

Lee S, Yang G, Yong Y, Liu Y, Zhao L, Xu J, Zhang X, Wan Y, Feng C, Fan Z, Liu Y, Luo J, Ke ZJ. ADAR2-dependent RNA editing of GluR2 is involved in thiamine deficiency-induced alteration of calcium dynamics. Mol Neurodegener. 2010; 5:54. [PubMed: 21110885]

Levine B, Klionsky DJ. Development by self-digestion: molecular mechanisms and biological functions of autophagy. Dev Cell. 2004; 6:463-477. [PubMed: 15068787]

Liu D, Ke Z, Luo J. Thiamine deficiency and neurodegeneration: the interplay among oxidative stress, endoplasmic reticulum stress, and autophagy. Mol Neurobiol. 2016

Luo J. Autophagy and ethanol neurotoxicity. Autophagy. 2014; 10:2099-2108. [PubMed: 25484085]

Meng Y, Yong Y, Yang G, Ding H, Fan Z, Tang Y, Luo J, Ke ZJ. Autophagy alleviates neurodegeneration caused by mild impairment of oxidative metabolism. J Neurochem. 2013; 126:805-818. [PubMed: 23586593]

Mizushima N, Klionsky DJ. Protein turnover via autophagy: implications for metabolism. Annu Rev Nutr. 2007; 27:19-40. [PubMed: 17311494]

Morishima N, Nakanishi K, Tsuchiya K, Shibata T, Seiwa E. Translocation of Bim to the endoplasmic reticulum (ER) mediates ER stress signaling for activation of cas-pase-12 during ER stressinduced apoptosis. J Biol Chem. 2004; 279:50375-50381. [PubMed: 15452118]

Ozcan L, Tabas I. Role of endoplasmic reticulum stress in metabolic disease and other disorders. Annu Rev Med. 2012; 63:317-328. [PubMed: 22248326]

Pannunzio P, Hazell AS, Pannunzio M, Rao KV, Butterworth RF. Thiamine deficiency results in metabolic acidosis and energy failure in cerebellar granule cells: an in vitro model for the study of cell death mechanisms in Wernicke's encephalopathy. J Neurosci Res. 2000; 62:286-292. [PubMed: 11020221]

Papandreou I, Denko NC, Olson M, Van Melckebeke H, Lust S, Tam A, Solow-Cordero DE, Bouley DM, Offner F, Niwa M, Koong AC. Identification of an Ire1alpha endonuclease specific inhibitor with cytotoxic activity against human multiple myeloma. Blood. 2011; 117:1311-1314. [PubMed: 21081713] 
Park LC, Albers DS, Xu H, Lindsay JG, Beal MF, Gibson GE. Mitochondrial impairment in the cerebellum of the patients with progressive supranuclear palsy. J Neurosci Res. 2001; 66:10281034. [PubMed: 11746433]

Perluigi M, Coccia R, Butterfield DA. 4-Hydroxy-2-nonenal, a reactive product of lipid peroxidation, and neurodegenerative diseases: a toxic combination illuminated by redox proteomics studies. Antioxid Redox Signal. 2012; 17:1590-1609. [PubMed: 22114878]

Perri ER, Thomas CJ, Parakh S, Spencer DM, Atkin JD. The unfolded protein response and the role of protein disulfide isomerase in neurodegeneration. Front Cell Dev Biol. 2016; 3:80. [PubMed: 26779479]

Radi E, Formichi P, Battisti C, Federico A. Apoptosis and oxidative stress in neurodegenerative diseases. J Alzheimers Dis. 2014; 42(Suppl 3):S125-S152. [PubMed: 25056458]

Rao VL, Mousseau DD, Butterworth RF. Nitric oxide synthase activities are selectively decreased in vulnerable brain regions in thiamine deficiency. Neurosci Lett. 1996; 208:17-20. [PubMed: 8731164]

Schroder M. Endoplasmic reticulum stress responses. Cell Mol Life Sci. 2008; 65:862-894. [PubMed: 18038217]

Schwab C, Steele JC, McGeer PL. Neurofibrillary tangles of Guam parkinson-dementia are associated with reactive microglia and complement proteins. Brain Res. 1996; 707:196-205. [PubMed: 8919296]

Tabas I, Ron D. Integrating the mechanisms of apoptosis induced by endoplasmic reticulum stress. Nat Cell Biol. 2011; 13:184-190. [PubMed: 21364565]

Todd K, Butterworth RF. Mechanisms of selective neuronal cell death due to thiamine deficiency. Ann N Y Acad Sci. 1999; 893:404-411. [PubMed: 10672278]

Troncoso JC, Johnston MV, Hess KM, Griffin JW, Price DL. Model of Wernicke's encephalopathy. Arch Neurol. 1981; 38:350-354. [PubMed: 7236062]

Wang X, Fan Z, Wang B, Luo J, Ke ZJ. Activation of double-stranded RNA-activated protein kinase by mild impairment of oxidative metabolism in neurons. J Neurochem. 2007a; 103:2380-2390. [PubMed: 17953670]

Wang X, Wang B, Fan Z, Shi X, Ke ZJ, Luo J. Thiamine deficiency induces endoplasmic reticulum stress in neurons. Neuroscience. 2007b; 144:1045-1056. [PubMed: 17137721]

Whitemarsh RC, Strathman MJ, Chase LG, Stankewicz C, Tepp WH, Johnson EA, Pellett S. Novel application of human neurons derived from induced pluripotent stem cells for highly sensitive botulinum neurotoxin detection. Toxicol Sci. 2012; 126:426-435. [PubMed: 22223483]

Xu X, Lei Y, Luo J, Wang J, Zhang S, Yang XJ, Sun M, Nuwaysir E, Fan G, Zhao J, Lei L, Zhong Z. Prevention of beta-amyloid induced toxicity in human iPS cell-derived neurons by inhibition of cyclin-dependent kinases and associated cell cycle events. Stem Cell Res. 2013; 10:213-227. [PubMed: 23305945]

Yang F, Luo J. Endoplasmic reticulum stress and ethanol neurotoxicity. Biomolecules. 2015; 5:25382553. [PubMed: 26473940]

Zhang K. Integration of ER stress, oxidative stress and the inflammatory response in health and disease. Int J Clin Exp Med. 2010; 3:33-40. [PubMed: 20369038] 
$\mathbf{A}$

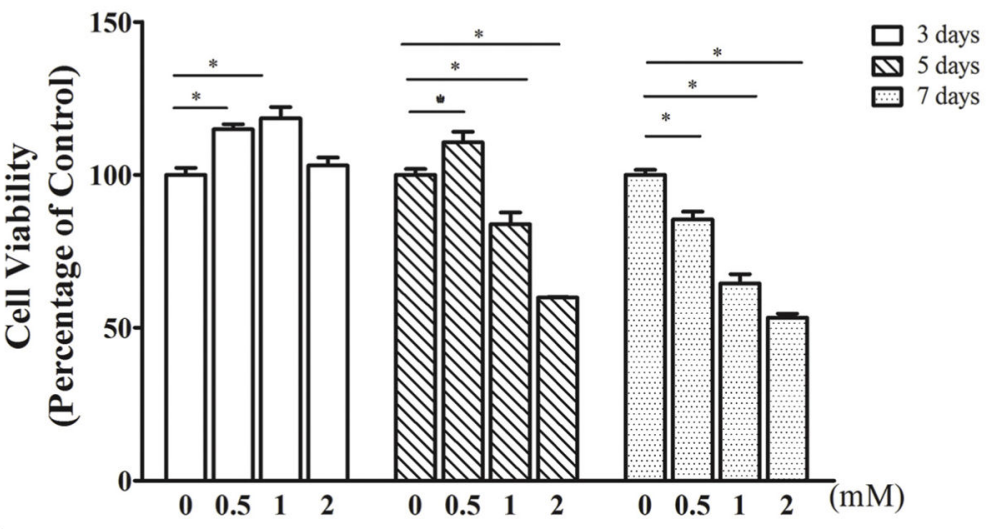

Control

TD

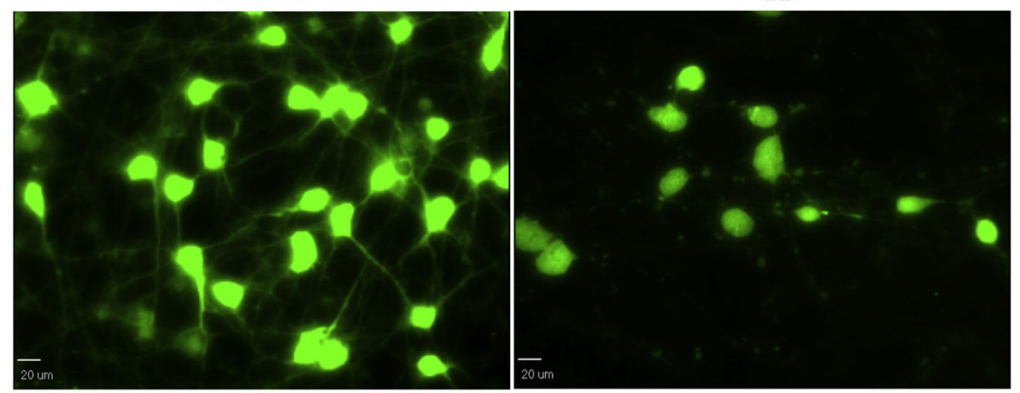

Fig. 1.

Effect of TD on the viability of cultured iCell neurons. A. The cultured iCell neurons were treated with amprolium (Amp: $0,0.5,1$, or $2 \mathrm{mM}$ ) for specified times, and the viability of cells was determined by CCK-8 assay as described under the Materials and methods. The experiment was replicated four times, and the results were expressed as the mean $\pm \mathrm{SEM} .{ }^{*} p$ $<0.05$, denote significant difference. B. The cultured iCell neurons were treated with amprolium (Amp: 0 or $1 \mathrm{mM}$ ) for 5 days. The neurons were stained with calcein AM and examined under the microscope. Bar $=20 \mu \mathrm{m}$. 
A

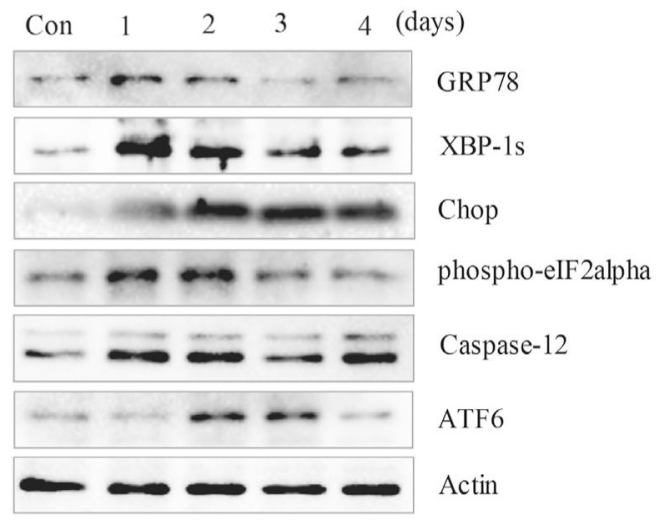

B

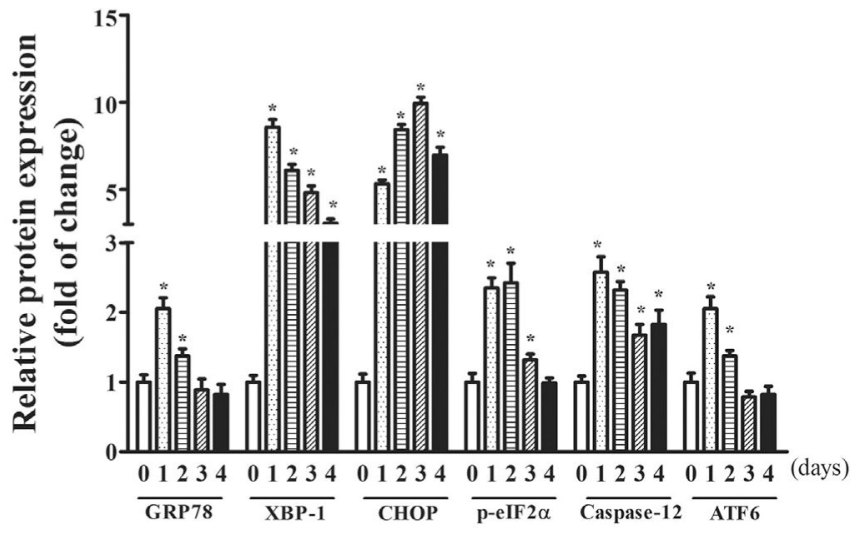

Fig. 2.

TD-induced ER stress in iCell Neurons. A. iCell neurons were treated with amprolium $(0$ or $1 \mathrm{mM}$ ). At specified times after the treatment, the lysates of the iCell neuron samples were collected. The expressions of GRP78, XBP-1, CHOP, p-eIF2a, caspase-12, and ATF6 were determined by immunoblotting. B. The relative amounts of GRP78, CHOP, p-eIF2a, cleaved caspase-12, and ATF6 were measured microdensitometrically and normalized to the expression of actin. The experiment was replicated three times, and the results were expressed as the mean \pm SEM. ${ }^{*} p<0.05$, statistically significant difference from untreated control groups. 


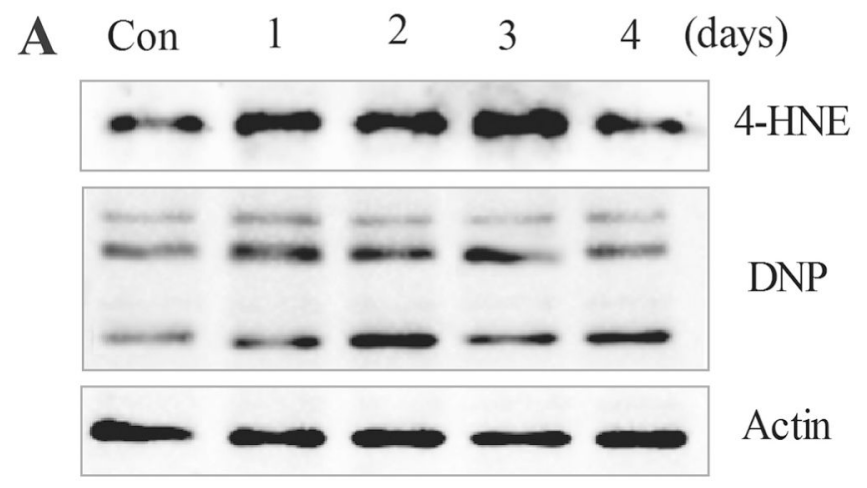

B

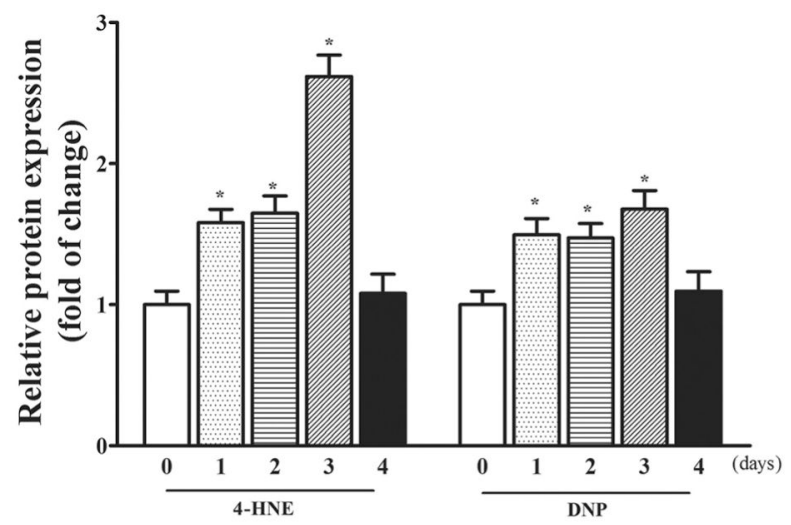

Fig. 3.

TD-induced oxidative stress in iCell Neurons. A. iCell neurons were treated with amprolium ( 0 or $1 \mathrm{mM}$ ) At specified times after the treatment, the lysates of iCell neurons samples were collected. The expressions of 4-HNE and DNP were determined by immunoblotting. B. The relative amounts of 4-HNE and DNP were measured microdensitometrically and normalized to the expression of actin. The experiment was replicated three times, and the results were expressed as the mean \pm SEM. ${ }^{*} p<0.05$, statistically significant difference from untreated control groups. 


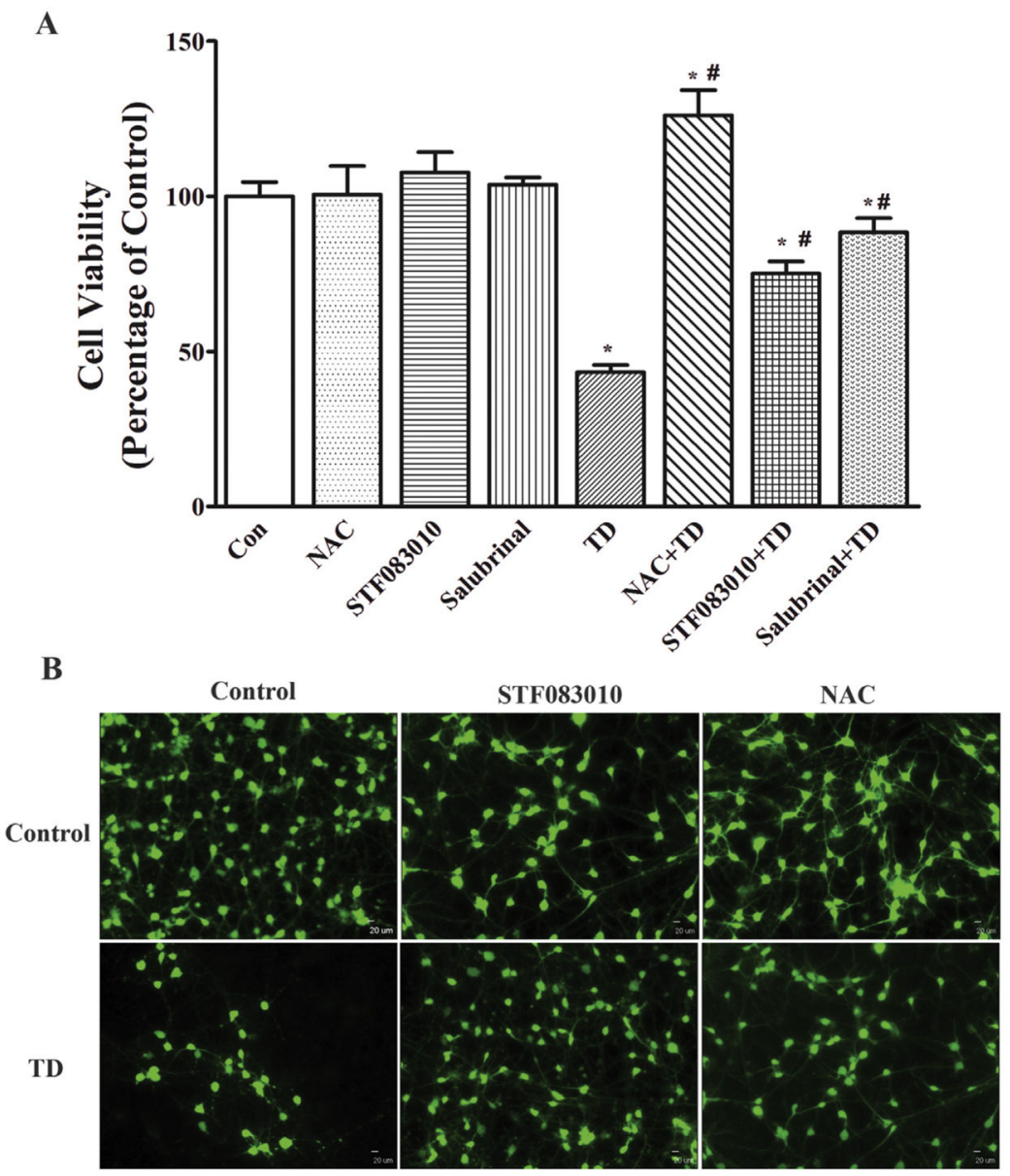

Fig. 4.

Inhibition of ER stress or oxidative stress protects iCell Neurons against TD-induced cell death. A. The cultured iCell Neurons were pretreated with STF083010 (10 $\mu \mathrm{M})$, Salubrinal $(10 \mu \mathrm{M})$, or NAC $(1 \mathrm{mM})$ for $1 \mathrm{~h}$, and followed by exposure to $1 \mathrm{mM}$ amprolium in the continued presence of STF083010, Salubrinal, or NAC for 5 days. The viability of cells was determined by CCK-8. The experiment was replicated three times, and the results were expressed as the mean \pm SEM. ${ }^{*} p<0.05$, statistically significant difference from untreated control groups. ${ }^{*} p<0.05$, statistically significant difference from amprolium alone groups. B. The cultured iCell neurons were pretreated with STF083010 (0 or $10 \mu \mathrm{M})$ or NAC (0 or 1 $\mathrm{mM}$ ) for $1 \mathrm{~h}$, and followed by exposure to $1 \mathrm{mM}$ amprolium in the presence or absence of STF083010 or NAC for 5 days. The neurons were stained with calcein AM and examined under the microscope. $\mathrm{Bar}=20 \mu \mathrm{m}$. 